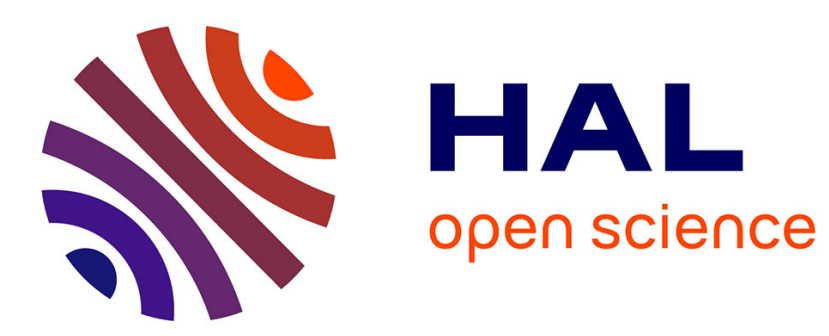

\title{
Traceologie fonctionnelle des matières osseuses : une méthode
}

\author{
Isabelle Sidéra, Alexandra Legrand-Pineau
}

\section{To cite this version:}

Isabelle Sidéra, Alexandra Legrand-Pineau. Traceologie fonctionnelle des matières osseuses: une méthode. Bulletin de la Société préhistorique française, 2006, tome 103 (n²), pp.291-304. 10.3406/bspf.2006.13434 . halshs-00076584

\section{HAL Id: halshs-00076584 \\ https://shs.hal.science/halshs-00076584}

Submitted on 26 May 2006

HAL is a multi-disciplinary open access archive for the deposit and dissemination of scientific research documents, whether they are published or not. The documents may come from teaching and research institutions in France or abroad, or from public or private research centers.
L'archive ouverte pluridisciplinaire HAL, est destinée au dépôt et à la diffusion de documents scientifiques de niveau recherche, publiés ou non, émanant des établissements d'enseignement et de recherche français ou étrangers, des laboratoires publics ou privés. 
Isabelle SIDÉRA et Alexandra LEGRAND

\title{
Tracéologie fonctionnelle des matières osseuses : une méthode
}

\begin{abstract}
Résumé
Les analyses fonctionnelles des assemblages osseux préhistoriques se sont considérablement multipliées cette dernière décennie. Mais pour autant, si un consensus commun existe, les méthodes ont encore aujourd'hui un caractère exploratoire et leurs résultats sont discutés. Il en existe plusieurs, toujours à l'épreuve, qui ne sont pas établies sur les mêmes critères d'observation ni sur les mêmes préalables théoriques. De ces différentes méthodes, nous en exposerons une ici, élaborée sur les industries en os, en bois de cerf et en ivoire du Néolithique, qui a déjà trouvé un écho au travers de travaux sur la parure en coquillage (Bonnardin, 2004). Cette méthode, qui provient d'une synthèse de quatre décennies de recherche, procède en deux temps complémentaires : une analyse des volumes alliée à une première analyse des surfaces, dite macroscopique, et une analyse approfondie des surfaces, dite microscopique. Nous sommes aujourd'hui, en ce qui concerne la tracéologie des matériaux non lithiques, face à une situation équivalente à celle qui prévalait pour la tracéologie du silex dans les années quatre-vingt : en cours de rationalisation et de discussion. Il y a donc urgence à exposer les méthodologies, afin d'élargir la discussion et de mettre en commun les savoirs.
\end{abstract}

\begin{abstract}
Use-wear analysis of prehistoric bone assemblages has increased considerably during the last 10 years. Despite an existing consensus regarding the methods used to date, they are still at an exploratory stage and their results the subject of debate. Several are still being tested and are based on different observational criteria and different preliminary theories. We will expound upon one of these different methods here; it was created when researching bone, antler and ivory industries of the Neolithic period, and has already been echoed in work carried out on shell ornaments (Bonnardin, 2004). This method stems from the synthesis of 40 years of research and has two complementary levels: 1) an analysis of volumes combined with a preliminary analysis of the surfaces, known as macroscopic, 2) a thorough analysis of surfaces, known as microscopic. We are, nowadays, facing a similar situation to the one prevailing in the 1980s regarding use-wear analysis of flint which underwent a process of restudy and discussion. Consequently, it is urgent to expound upon methodologies in order to broaden debate and pool knowledge.
\end{abstract}

L'étude systématique des objets en matière osseuse et en coquille est récente puisqu'elle débute dans les années soixante avec les travaux de Henriette Camps-
Fabrer (1966) puis d'Yvette Taborin (1993). Si la fonction des objets a été envisagée dès les premières études, la tracéologie en tant que telle émerge 
seulement à la fin des années soixante-dix. S'appuyant sur les recherches avant-gardistes de S.A. Semenov (1964), c'est Danielle Stordeur qui en fait les premiers développements en France (1983, avec AndersonGerfaut, 1985). Nous pouvons donc nous targuer aujourd'hui d'une tradition d'étude de l'outillage osseux longue de 40 ans et plus, modernisée dans les années soixante-dix. Des approches différentes, liées aux personnalités des chercheurs investis dans cette recherche thématique, se sont superposées. Cette suite aboutit aujourd'hui à des protocoles d'étude implicites qu'il convient de clarifier. Quels sont les objectifs et les résultats de l'analyse fonctionnelle relative aux matériaux non lithiques? Quels sont les moyens que se donnent les tracéologues pour identifier et répondre au problème du décodage de la fonction matérielle des objets archéologiques? Quels sont les critères d'observation à hiérarchiser et selon quel protocole d'analyse? Où en est-on aujourd'hui des procédures et des résultats? Quelles sont les conditions de mise en œuvre et les limites des méthodes employées? Pour traiter ces questions, nous nous appuierons sur des exemples issus de l'étude de 10000 objets du Néolithique et du Chalcolithique européens, de la Bulgarie à la France, en y incluant ceux du Néolithique précéramique de Chypre. Ajoutons que la variété des contextes chronoculturels des industries est une source d'information et d'expérience enrichissante et nécessaire. Dans cette perspective, l'étude de nouvelles séries doit être constante. C'est au prix de ce renouvellement permanent que nous cernerons davantage la diversité comme la répétition des traces et que nous progresserons dans leur appréhension.

\section{EXPÉRIMENTATION}

(fig. 1)

L'expérimentation est un préambule indispensable car elle apporte un secours à la compréhension de l'origine des stigmates des objets archéologiques, des mécanismes d'usure et des réponses des matériaux et des types d'outils définis, aux conditions de travail qui leur sont imposées. Elle conforte, en outre, la recherche tracéologique. Dans les pas des technologues et des tracéologues de la pierre taillée (Pelegrin, 1991; Plisson, 1991; Anderson, 1992 ; Christensen, 1996), la pratique expérimentale, mise en œuvre avec une certaine systématique depuis plus de 20 ans, concerne les artefacts osseux. Malgré toutes ces années de pratique, les référentiels sont cependant, aujourd'hui encore, très partiels. C'est donc à les compléter qu'il convient de travailler.

Commençons par un bilan historique. Les expérimentations les plus nombreuses ont documenté les techniques (Poplin, 1974; Dauvois, 1974; Billamboz, 1977 ; Camps-Fabrer et d'Anna, 1977 ; Newcomer, 1977 ; Stordeur, 1977; Barge, 1982; Ettos, 1985 ; Sénépart, 1991; Nandris et Camps-Fabrer, 1993; Sidéra, 1993a et 2001; Chiquet et al., 1997; David, 1999 et 2003; Liolios, 1999; Provenzano, 1999; Averbouh, 2000 ; Maigrot, 2001; Schibler, 2001 ; Goutas, 2004; Pétillon, 2004; Legrand, 2005a et b). Ainsi, la superposition de ces expérimentations conduit à une bonne connaissance des techniques et des méthodes de fabrication des artefacts osseux (Ramseyer dir., 2005) et à un progrès réel en matière de technologie culturelle (Sidéra, 2005). En revanche, les résultats relatifs à l'aspect fonctionnel sont moindres. Certains types d'outils seulement sont bien définis tels des égrenoirs, des racloirs de potiers, des pendeloques, des écharnoirs, des palissons, des ciseaux, des chasse-lame et des retouchoirs, des couteaux à glace et des haches en bois de cerf (Stordeur et Anderson-Gerfaut, 1985; Peltier, 1986; Peltier et Plisson, 1986; Campana, 1989; Ettos, 1991; d'Errico, 1993; d'Errico et al., 1995; Meneses Fernandez, 1993; Sidéra, 1993a; Lemoine, 1997; Maigrot, 1997, 2001 et 2003; Christidou, 1999 et 2001; Legrand, 2000; Averbouh et Bodu, 2002; Sidéra et Giacobini, 2002; Martineau et Maigrot, 2004; Christidou et Legrand, 2005). Ces expérimentations sont des plus nécessaires pour fiabiliser les attributions fonctionnelles à terme, mais manquent encore de systématique et de variété. La compréhension de l'origine de l'usure, ses mécanismes
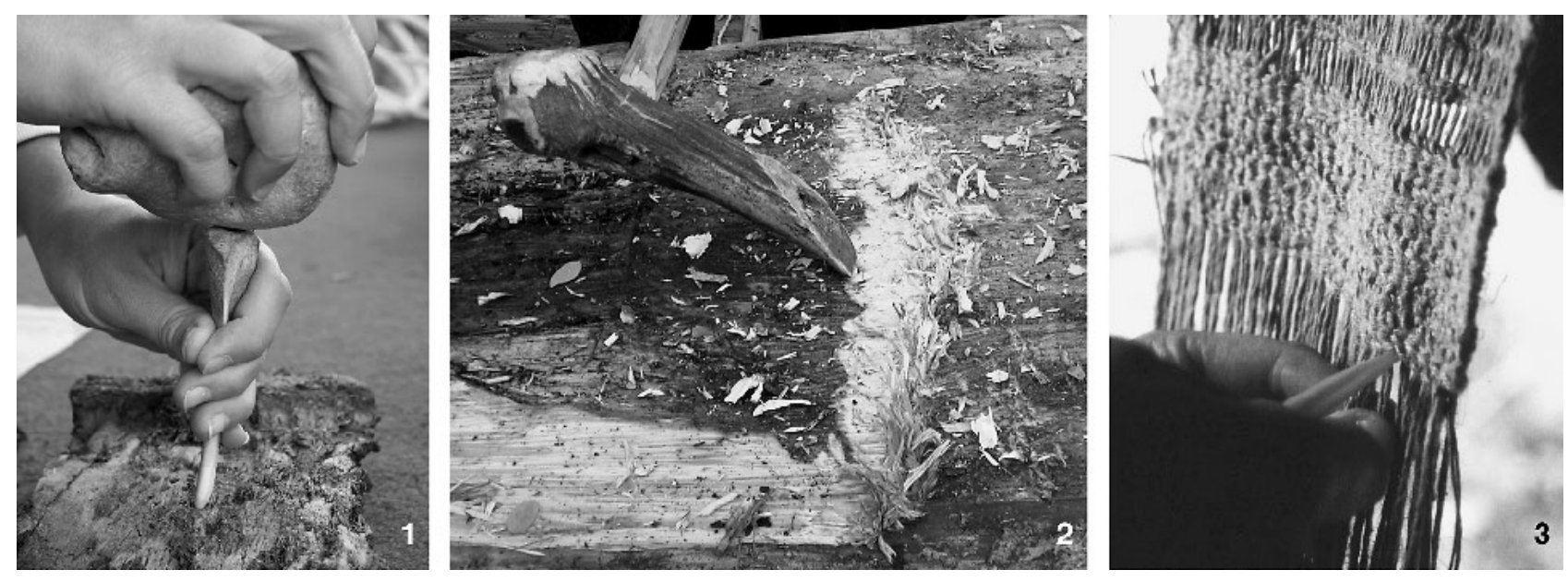

Fig. 1 - $\mathrm{N}^{\circ} 1$ : perforation de l'écorce avec un poinçon par percussion indirecte; $\mathrm{n}^{\circ} 2$ : creuser du bois avec une hache en bois de cerf; $\mathrm{n}^{\circ} 3:$ tasser des fibres végétales avec un poinçon. $\mathrm{N}^{\text {os }} 1$ et 3 : expérimentation et cliché A. Legrand; $n^{\circ} 2$ : expérimentation et cliché I. Sidéra. 
de formation et ses spécificités, en relation avec une action, une matière, un état de celle-ci, une morphologie d'objet, etc., est essentielle dans ce projet. En outre, l'accroissement des connaissances complexifie le problème de l'identification fonctionnelle. Nous savons aujourd'hui, grâce à une succession de travaux, que le poli n'est pas la manifestation la plus diagnostique d'une usure donnée (Christidou, 1999). À la différence de la pierre, le poli de l'os n'est pas représentatif de la matière travaillée, sauf exception. Il ne s'agit donc pas de chercher à acquérir différents types de polis en travaillant plusieurs matières selon une durée courte et déterminée, mais de mettre en œuvre l'usure de façon plus générale. Nous savons également que la matière osseuse a été utilisée à différents états : fraîche, sèche ou chauffée (Stordeur, 1988; Sénépart, 1991; Sidéra, 2001). L'état de l'os, qui conditionne l'enregistrement des traces, est donc une variable qui s'ajoute aux autres. Sans l'avoir jamais réellement mesuré, on sait que les techniques de fabrication et les outils employés (lames retouchées, éclats bruts), leur nature (silex, quartz, grès, métal, etc.) influencent l'enregistrement des traces fonctionnelles (Christidou, 1999 et 2004 ; Christidou et Legrand, 2005 ; Cristiani et Alhaique, 2005). L'intégration de ce nouveau paramètre est donc aussi indispensable pour élaborer de nouvelles expérimentations. Le temps d'utilisation, qui est un paramètre également important, est un problème qui se pose avec pertinence sur les matières osseuses car les objets ont un usage long mais d'une durée indéfinie. Or si l'observation d'objets ethnographiques, qui sont aussi des pièces longuement utilisées, permet de contourner en partie ce problème, l'acquisition par l'expérimentation de pièces longuement ou très longuement usées reste une difficulté. Aujourd'hui seulement, par exemple, nous disposons de pièces sur lesquelles le travail a commencé il y a dix ans.

Tenant compte des paramètres qui viennent d'être évoqués, la somme des expérimentations nécessaires réclame donc un investissement considérable. Pour comprendre la variabilité de l'usure, il faut, plus que jamais, étendre rapidement l'expérimentation à une très large diversité de paramètres et intensifier la coopération entre expérimentateurs. De plus, il convient d'objectiver et de systématiser l'expérimentation en l'instrumentalisant. En effet, l'automatisation des usures, qui est notre projet actuel, paraît un moyen sûr, et pour progresser dans une compréhension mécanique et tribologique des traces d'utilisation, et pour mieux estimer le facteur du temps dans la formation et le développement de l'usure.

Ceci explique la contribution expérimentale que nous nous sommes donnée à réaliser. Elle consiste à accumuler la plus grande variété de types d'outils, mis en relation avec la plus grande diversité de matériaux travaillés. Les variations du temps d'utilisation, des techniques de fabrication, de l'état de la matière osseuse et des matériaux d'œuvre sont inclus dans les registres. L'accumulation est le seul moyen d'approcher la fonction matérielle du plus grand nombre d'objets, autrement dit de parvenir au référentiel tracéologique le plus complet possible. Dans cet optique,

\begin{tabular}{|c|c|c|}
\hline Matière & 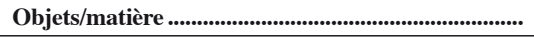 & Nbre \\
\hline \multirow{22}{*}{$\begin{array}{l}\text { Os de grands } \\
\text { mammifères }\end{array}$} & 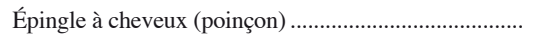 & 1 \\
\hline & 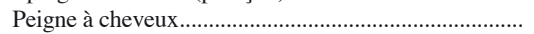 & 1 \\
\hline & Poinçon utilisé en percussion directe & \\
\hline & et indirecte sur peaux ............................... & 20 \\
\hline & Poinçon utilisé en percussion directe & \\
\hline & et indirecte sur diverses matières végétales ...................... & 13 \\
\hline & Poinçon utilisé en percussion directe sur os .................... & 2 \\
\hline & 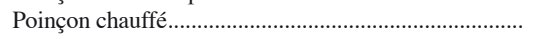 & 2 \\
\hline & 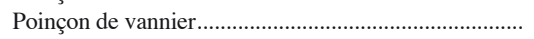 & 6 \\
\hline & Grattoir à peau (écharnoirs et palissons) ............................. & 22 \\
\hline & 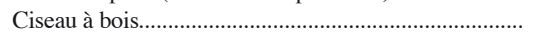 & 18 \\
\hline & 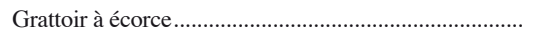 & 2 \\
\hline & 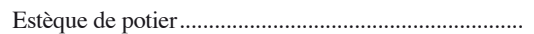 & 1 \\
\hline & Plaquette perforée à l'archet et au perçoir manuel........ & 2 \\
\hline & Un peigne à carder le lin composé de quatre dents...... & 1 \\
\hline & 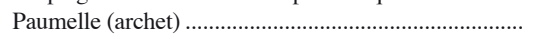 & 1 \\
\hline & 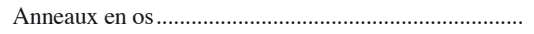 & 5 \\
\hline & Crochet boucle de ceinture & 1 \\
\hline & 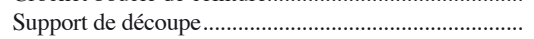 & 1 \\
\hline & 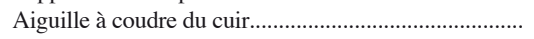 & 3 \\
\hline & 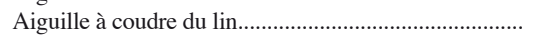 & 3 \\
\hline & 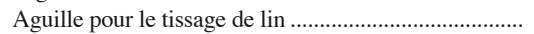 & 2 \\
\hline \multirow[t]{5}{*}{ Os d'oiseau } & 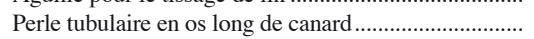 & 3 \\
\hline & 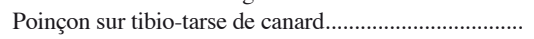 & 1 \\
\hline & Poinçon subactuel en os épissoir à maïs............................... & 1 \\
\hline & Poinçon subactuel de Papouasie-Nouvelle-Guinée & \\
\hline & 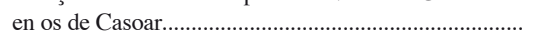 & 2 \\
\hline \multirow[t]{2}{*}{ Os de poisson } & Collier court de perles de vertèbres de poisson brutes. & 1 \\
\hline & Collier long de perles de vertèbres de poissons polies. & 1 \\
\hline \multirow[t]{11}{*}{ Bois de cerf } & 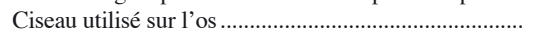 & 1 \\
\hline & 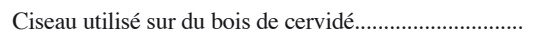 & 3 \\
\hline & 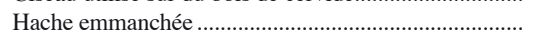 & 3 \\
\hline & 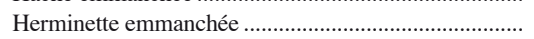 & 1 \\
\hline & 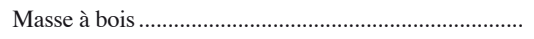 & 1 \\
\hline & 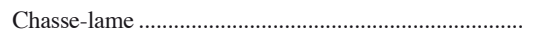 & 2 \\
\hline & 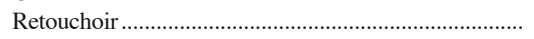 & 1 \\
\hline & 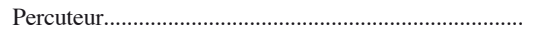 & 1 \\
\hline & 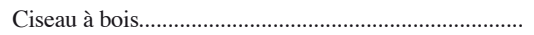 & 9 \\
\hline & 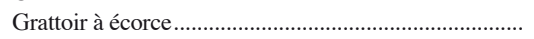 & 2 \\
\hline & 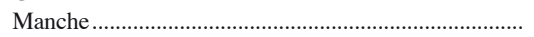 & 3 \\
\hline \multirow[t]{4}{*}{ Ivoire } & Bracelet subactuel de Nouvelle-Calédonie & \\
\hline & 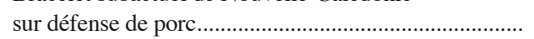 & 1 \\
\hline & Racloir à arc subactuel de Papouasie & \\
\hline & 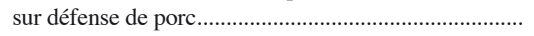 & 1 \\
\hline \multirow[t]{3}{*}{ Coquillages } & Collier court de petits coquillages & \\
\hline & type Nucella ou cauris.............. & 3 \\
\hline & & 148 \\
\hline
\end{tabular}

Tableau 1 - Liste de la collection d'objets expérimentaux et ethnographiques.

les auteurs de cet article ont réuni, à la maison de l'Archéologie et de l'Ethnologie René Ginouvès de Nanterre, une collection pour l'instant composée de 148 outils, objets mobiliers et parures, issus de contextes ethnographiques bien définis et testés au travers d'expériences individuelles ou de programmes collectifs (tabl. 1). Notre projet consiste à mettre notre collection de référence à la disposition des chercheurs. Nous espérons encore être rejointes par d'autres chercheurs dans cette démarche. Ici, la coopération est en effet indispensable, d'autant que l'approche fonctionnelle est de plus en plus courante. Il convient à notre sens de structurer et d'enrichir collectivement une collection de comparaison existante.

\section{APPROCHE FONCTIONNELLE}

Depuis les années quatre-vingt, les études fonctionnelles les plus nombreuses ont visé à comprendre la fonction de certains types d'outils ou à décrire les 


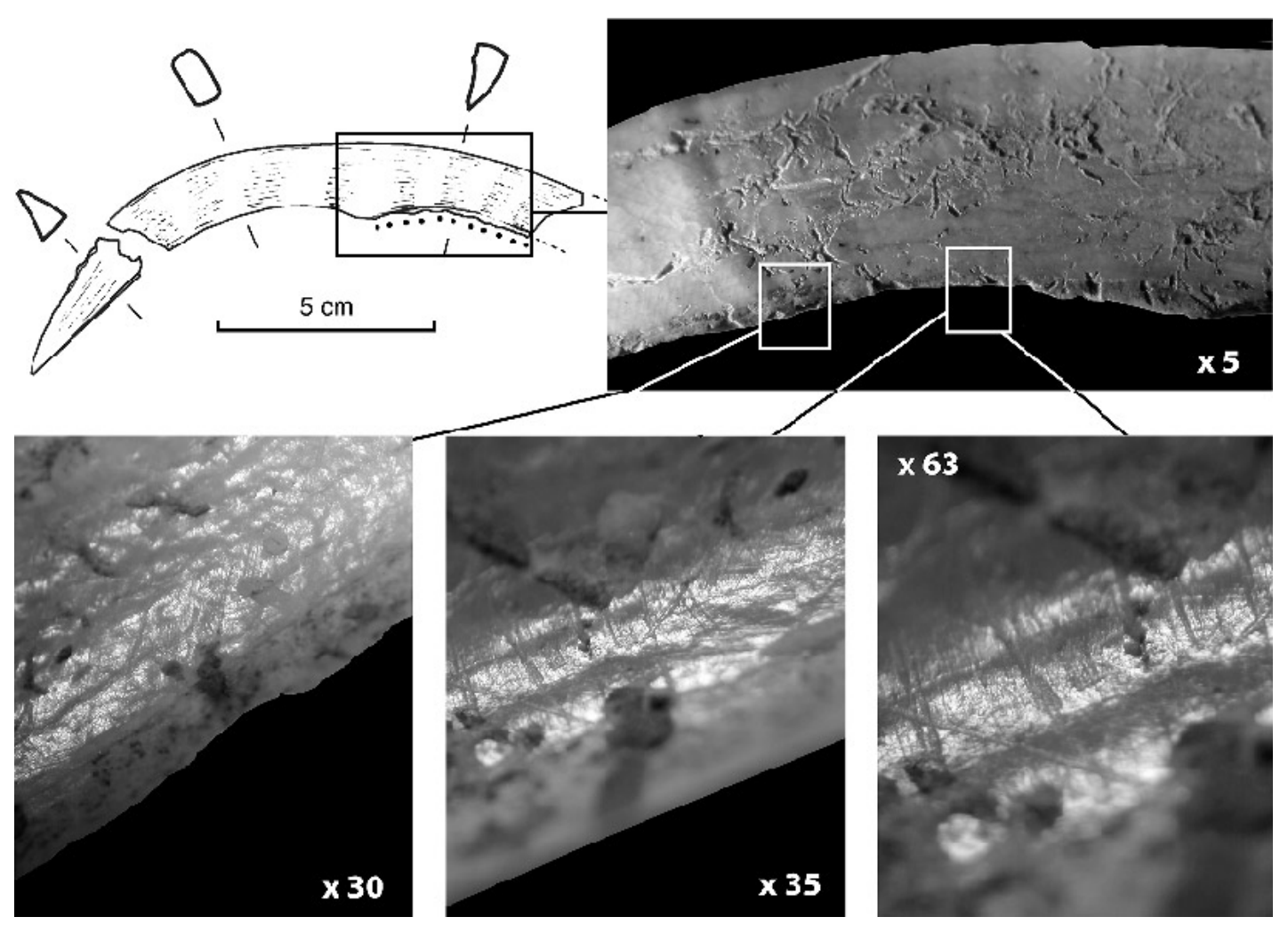

Fig. 2 - Défense de suidé archéologique de Mareuil-les-Meaux (Seine-et-Marne, fouilles R. Cottiaux, INRAP) (VSG). Grossissement progressif de la partie active de l'outil, face émaillée. À l'œil nu et à un grossissement de $5 \times$, voir l'apparence concave et ébréchée du bord actif. De $35 \times$ à $63 \times$, stries nombreuses et bien développées sur le bord actif, croisées et perpendiculaires. Un microémoussé apparaît sur le bord. Dessins et clichés I. Sidéra. D’après Sidéra, 2003.

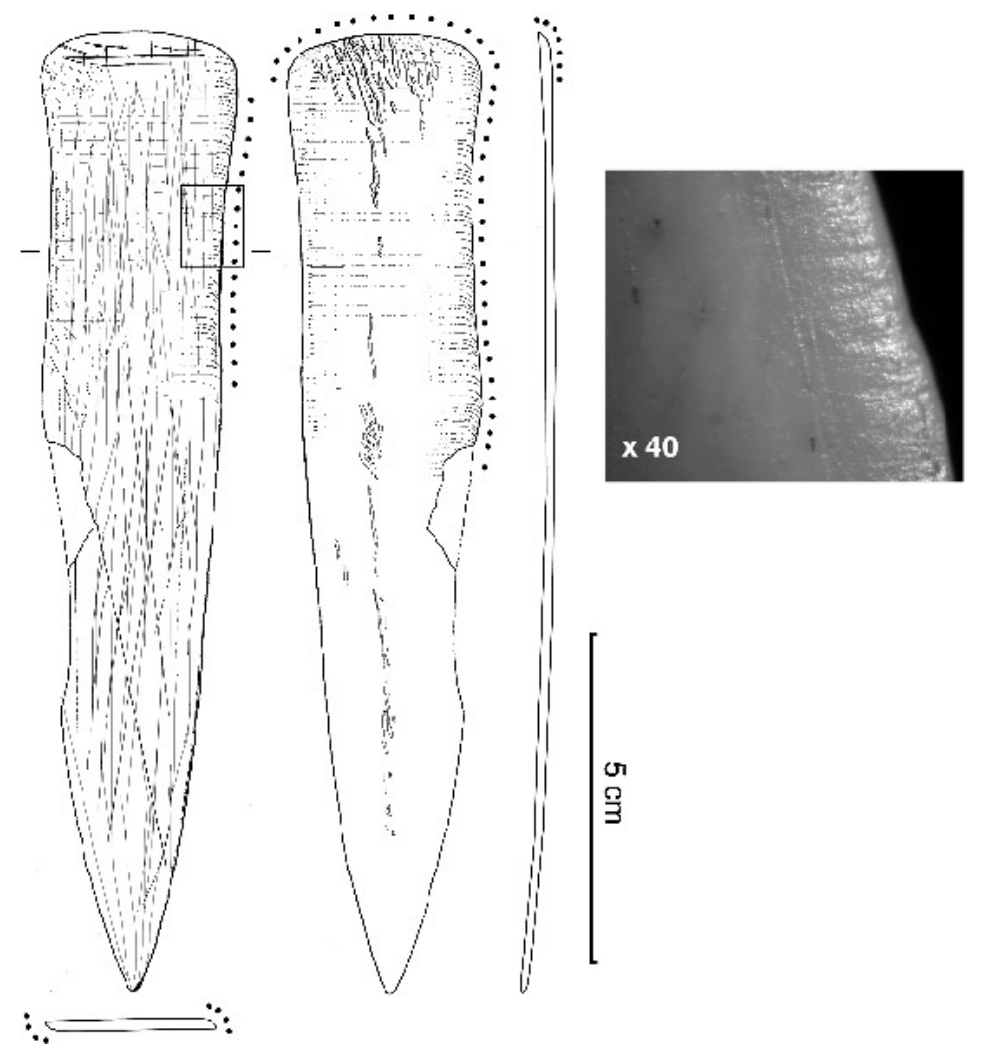

Fig. 3 - Racloir de potier archéologique de Corbères-les-Cabanes, grotte de Montou (Pyrénées-Orientales, fouilles F. Claustre, CNRS) (Montbolo). À un grossissement de $40 \times$, des stries nombreuses, larges, longues et rectilignes apparaissent perpendiculairement au bord actif. Dessins et clichés I. Sidéra. D'après Sidéra et Claustre, sous presse. 
altérations, qu'elles soient anthropiques ou taphonomiques (Stordeur, 1983; Stordeur et AndersonGerfaud, 1985; Olsen, 1989 et 2001; Ettos, 1991 ; d'Errico, 1991, 1993 et 1996; d'Errico et Giacobini, 1985 ; d'Errico et al., 1995 ; Meneses Fernandez, 1993 et 1994; d'Errico et Villa, 1997; Aimar et al., 1998; Legrand, 2003). Les études qui tendent à embrasser l'ensemble des objets d'une série archéologique définie sont moins nombreuses (Sordeur, 1988; Campana, 1989; Sidéra, 1989 et 1993a ; Lemoine, 1997; Christidou, 1999; Maigrot, 2003). Différents moyens d'observation sont donnés à l'approche fonctionnelle : soit le faible grossissement, par le biais d'un stéréomicroscope, soit le fort grossissement, par celui d'un microscope optique à réflexion (Stordeur, 1989). En réalité, il en existe d'autres, mais nous nous focaliserons ici sur notre démarche, qui a l'avantage d'employer un équipement courant. Le faible et le fort grossissement sont deux échelles d'observation complémentaires et interactives, car elles n'apportent pas nécessairement les mêmes informations.

\section{Altérations des volumes : examen macroscopique}

Selon un protocole d'examens graduels, qui commence à faire l'objet d'un consensus, la première étape est relative aux caractères dits macroscopiques, c'està-dire discernables à l'œil nu et au stéréomicroscope surtout. Les grossissements habituels s'étagent entre $10 \times$ et $80 \times$. Ils peuvent monter jusqu'à $130 \times$ (Maigrot, 2003). L'examen macroscopique est pertinent sur les matières osseuses et les coquilles car, plus encore que la pierre, les traces d'usage sont des empreintes immédiatement accessibles.

Le principe de l'examen macroscopique est de travailler sur le volume des pièces, dans une perspective mécanique (Sidéra, 1989 et 1993a). L'étude des surfaces peut également commencer à cette échelle, mais le microscope fournit une meilleure qualité d'observation. Ce ne sont d'ailleurs pas les mêmes informations que l'on recherche à l'une ou à l'autre échelle de grossissement. La justification théorique du travail sur les volumes est la suivante : les propriétés plastiques de l'os et sa moindre dureté comparée à la pierre conduisent à modifier de façon caractéristique, selon le travail effectué, le «volume initial de la pièce » (Sidéra, 1993a, p. 206-207). Il s'agit donc, par référence à une pièce qui vient d'être fabriquée, dont on a idée par les techniques, par les procédés de fabrication employés et par le faible degré d'usure d'autres pièces semblables, de circonscrire et de caractériser toutes les déformations qui résultent de l'usure, étant entendu que le raffûtage est inclus dans l'usure. La morphologie de cette modification du volume ou «déformation du modelé initial» est caractéristique de l'usage de la pièce.

Stries, polis ou colorations, enlèvements ou ébréchures apparaissent dès les premières minutes de l'emploi d'un objet donné, selon le travail exécuté (d'Errico, 1993 ; Sidéra, 1993a). Plus tard, l'émoussé apparaît et se développe, en déformant le contour, les pans du biseau, les pointes ou les profils des pièces initiales. En outre, le raffûtage qui se superpose à la zone usée accentue la déformation. Celle-ci est alors tangible et peut se décrire et se mesurer à l'échelle macroscopique. La combinaison usure et raffûtage - le raffûtage se superpose toujours presque exactement à l'usure et l'accentue - reflète plus ou moins clairement l'action de l'outil, la nature et la morphologie du matériau traité. Par exemple, à cause d'un bord concave, d'une extension limitée de leur bord actif, les racloirs, qui ont travaillé des matériaux étroits et bombés comme l'os ou le bois, peuvent être identifiés aisément; nous connaissons des exemples ethnographiques en Papouasie-Nouvelle-Guinée (fig. 2) (Sidéra, 1993a et 2000). C'est la perte de matière due à l'usure et surtout au raffûtage opéré pour garder un tranchant vif qui aboutit à la concavité. La délinéation concave du bord actif est aussi caractéristique des estèques (fig. 3), comme elle matérialise la course de certaines pointes (fig. 4). Les mêmes mécanismes d'usure sont à l'œuvre, sauf pour les racloirs de potiers, où seul le frottement sur une matière globuleuse et abrasive conduit à une perte de matière équivalente (Martineau et Maigrot, 2004 ; Sidéra, 1993a; Sidéra et Claustre, à paraître).

En fonction de différents mécanismes, la matière osseuse finit en tout cas par épouser, en miroir, la morphologie de la matière traitée. C'est pourquoi les déformations du modelé initial sont diagnostiques du point de vue fonctionnel, comme le sont aussi, et pour les mêmes raisons, celles du microprofil des outils tranchants, que l'on peut apprécier à un grossissement de $20 \times$ à $80 \times($ fig. 5). Citons, pour illustrer ce propos, un exemple issu de l'outillage rubané de Cuiry-lèsChaudardes (Aisne). Sur ce site, deux types de grattoirs en os coexistent. Leur facture est très similaire, à l'exception de la forme de chacun de leur tranchant (fig. 6) (Sidéra, 1989 et 1993b). L'un est un biseau double aux pans courts et plats, affûté, avec de très nombreuses et courtes stries, rectilignes et larges, laissées par un raffûtage fréquent (fig. 6, $n^{\circ} 1$ ). Le second est un biseau simple, dont la face de contact est courbe, émoussée et lustrée, avec de longues stries, fines et courbes (fig. 6, $n^{\circ} 4$ ). Le premier instrument était, d'après l'expérimentation et la correspondance des traces, utilisé en frottement linéaire et continu, pour écharner des peaux fraîches, probablement disposées sur un billot (fig. 6, A). Le second était utilisé en frottement discontinu dans un mouvement de pendule, durant une étape postérieure du travail de la peau, probablement pour assouplir la peau tendue sur un cadre (fig. 6, B). Dans les deux cas, la morphologie du biseau ne résulte pas seulement de l'usure. Elle est déterminée au façonnage et entretenue par le raffûtage, dans le respect de la forme originale (fig. 6, $\mathrm{n}^{\circ} 1$ ). D'ailleurs, le second type n'est pratiquement jamais raffûté. Cet exemple illustre aussi la complexité des interactions entre le façonnage, l'usure et le raffûtage, qui demandent, pour être comprises, une interprétation fonctionnelle complète englobant la compréhension du mécanisme de l'usure. S'ajoute alors une autre 


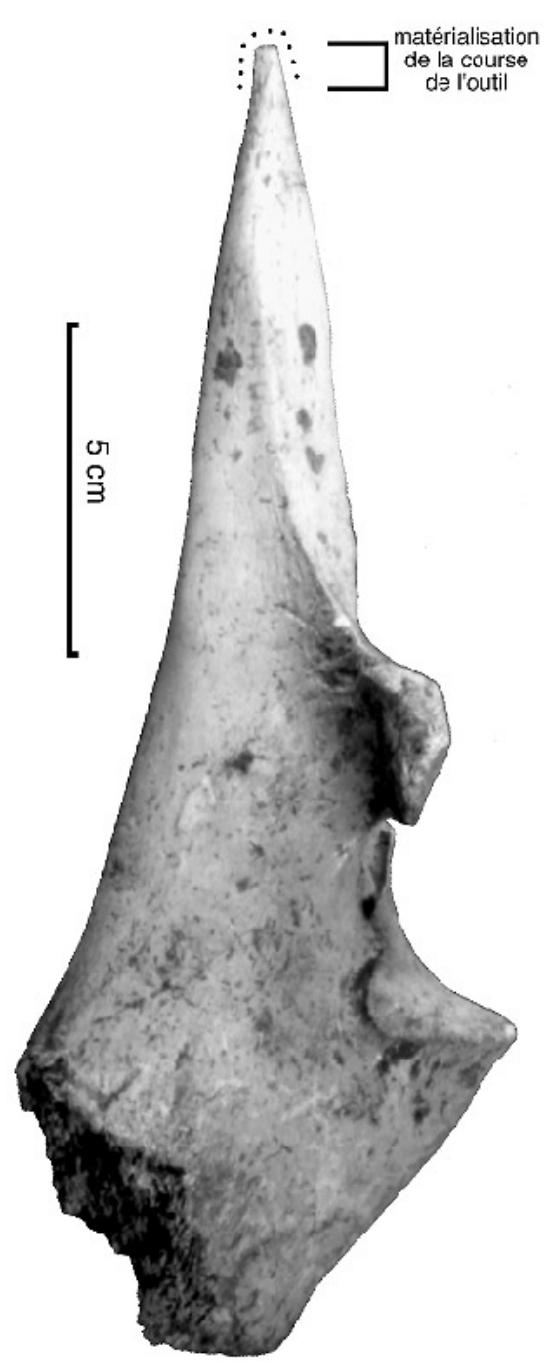

Fig. 4 - Poinçon archéologique de Cuiry-lès-Chaudardes (Aisne, France, ERA 12 excavations) (RRBP). Déformation de la pointe due à l'usage et au raffûtage, qui matérialise la course de l'outil. Cliché S. Ouboukoff. méthode, dont l'application est possible si l'on dispose d'une grande série : la reconstitution de la chaîne d'usure (Sidéra et Giacobini, 2002). Il convient de chaîner les degrés d'usure, du plus faible au plus fort, pour chaque type d'outils. Ainsi est-il permis de visualiser la progression de l'usure d'un outil à l'autre, d'en mesurer le développement, du départ à l'arrivée, c'està-dire à l'abandon des pièces parvenues entières au terme de leur processus d'usure (stade terminal d'usure). Cette méthode a été mise au point sur la série d'outils très fournie de Drama (Bulgarie) (Sidéra, 1997 et non publié) et appliquée ensuite à une série de perles funéraires (fig. 7). Le chaînage de l'usure a fait apparaître la constitution évolutive de la parure, qui est sans cesse complétée par des perles neuves ou presque qui côtoient de plus anciennes (Sidéra et Giacobini, 2002). La même méthode, encore appliquée à un vaste corpus d'objets de parure de coquillage, a permis de tirer les résultats les plus dynamiques sur le mode de constitution des parures funéraires (Bonnardin, 2004). Le principal apport de ces méthodes, qui répondent d'une étude de la mécanique de l'usure, est le caractère anthropologique de l'information qu'elles fournissent.

Les déformations du modelé initial sont souvent accompagnées par d'autres altérations de volume, toujours aussi prégnantes, tels différents types d'enlèvements, d'arrachements, d'écrasements et d'émoussés, et des altérations de surface comme les polis et les stries, que nous pouvons aussi en partie scruter au stéréomicroscope (fig. 8). En définitive, les altérations de volume et de surface ne doivent pas être dissociées. Pour mieux expliquer notre démarche, reprenons l'exemple des racloirs cités plus haut. S'ils ont en commun des bords concaves issus d'un processus d'usure proche, leurs différences majeures résident dans la présence d'ébréchures jusqu'à de vrais enlèvements sur le bord actif des racloirs à bois et à os. Les racloirs de potier sont caractérisés par un émoussé arrondi de leurs bords et des surfaces de contact adjacentes. Cet émoussé est très marqué et croisé de stries
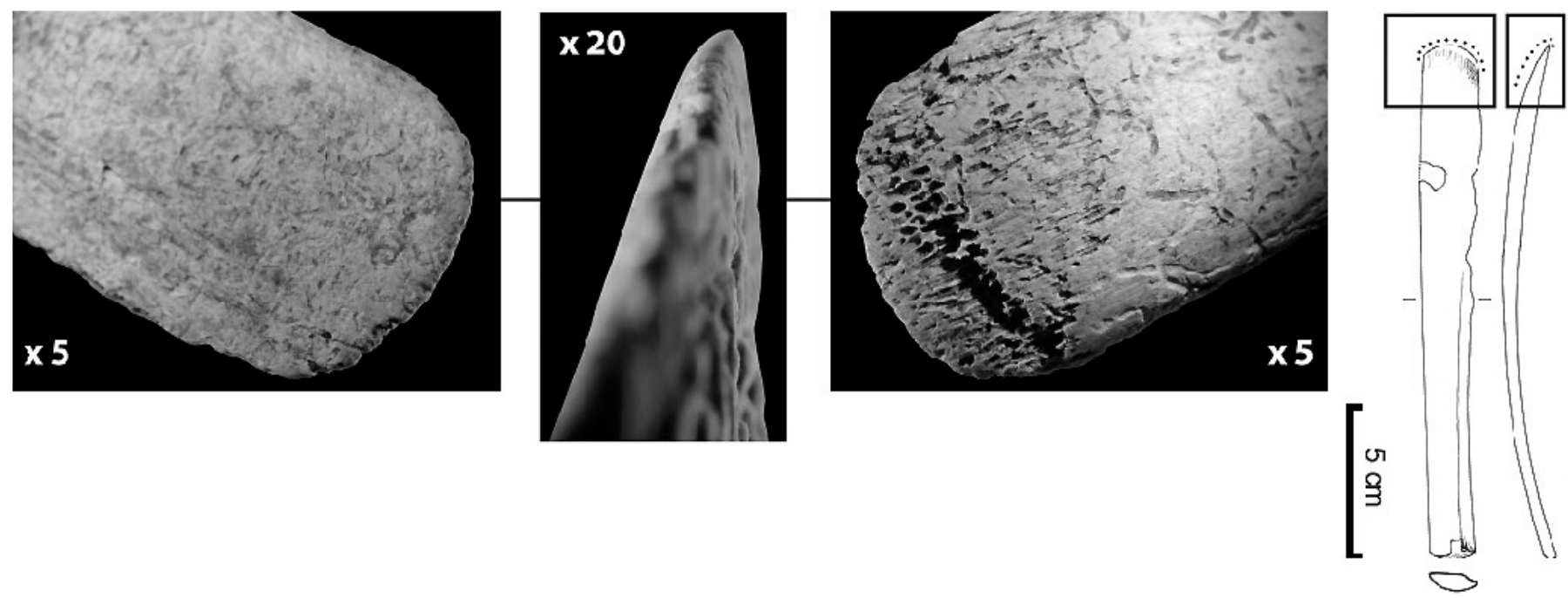

Fig. 5 - Écharnoir archéologique de Mareuil-les-Meaux (Seine-et-Marne, fouilles R. Cottiaux, INRAP) (VSG). Faces supérieure, inférieure et profil. Voir la morphologie du profil du fil du tranchant, caractérisée par un microbiseau émoussé symétrique. Le taillant est aigu. Dessin et clichés I. Sidéra. D'après Sidéra, 2003. 


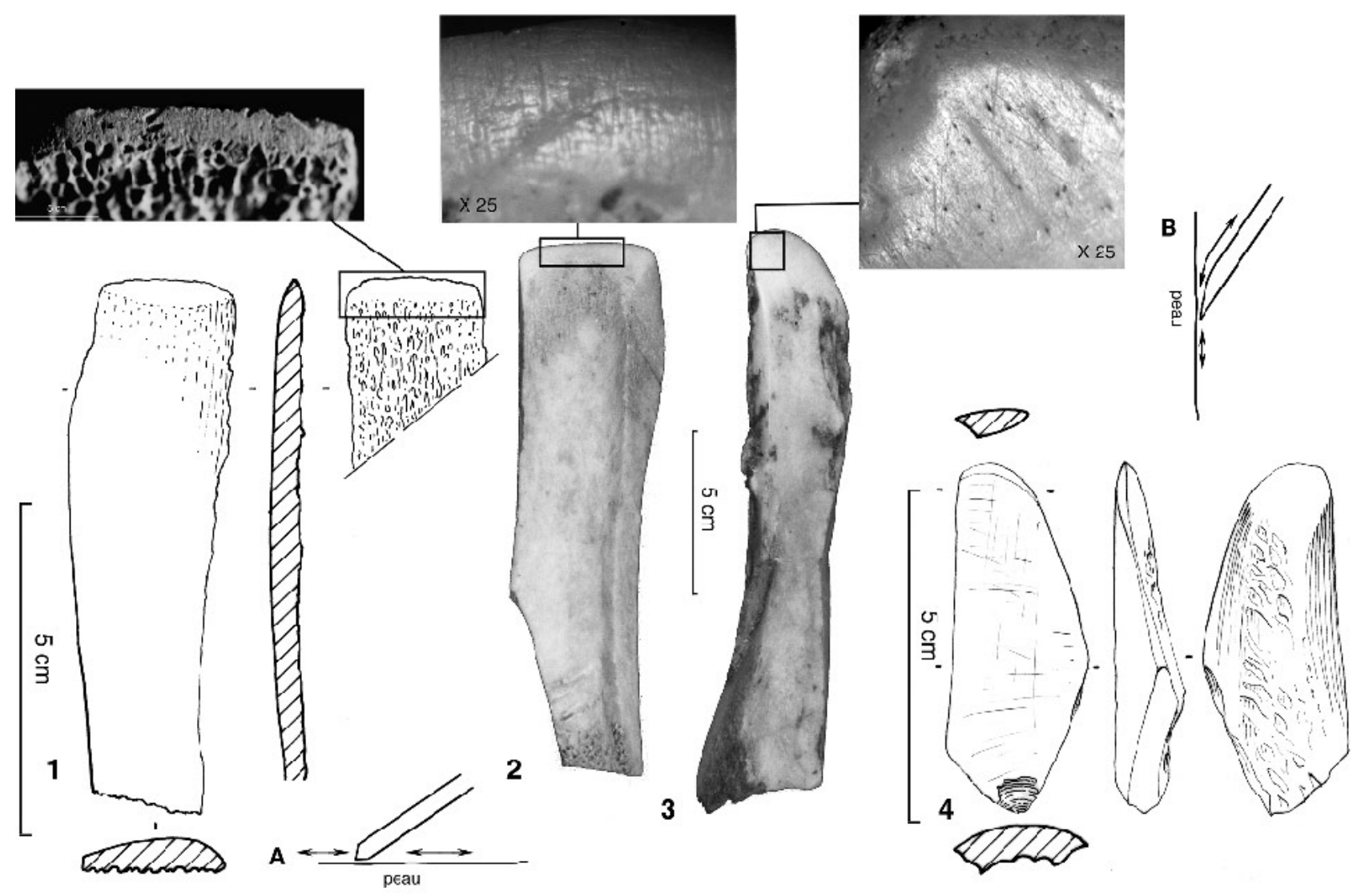

Fig. 6 - Grattoirs à peau archéologiques de Cuiry-lès-Chaudardes (Aisne, fouilles ERA 12) et expérimentaux. N 1 : écharnoir archéologique sur côte de grand ruminant, type 1 , double biseau à pans plats, avec un taillant aigu et de nombreuses stries dues au raffûtage par abrasion; $\mathrm{n}^{\circ} 2$ : réplique expérimentale et usée d'un écharnoir de type 1 avec des stries courtes, larges et rectilignes sur la partie active dues au raffûtage et à l'utilisation d'un abrasif; $n^{\circ} 3$ : réplique expérimentale et usée d'un palisson avec des stries longues et courbes; $n^{\circ} 4:$ modèle archéologique du palisson de type 2 , de Cuiry-lèsChaudardes; A : mode d'utilisation de l'écharnoir de type 1; B : mode d'utilisation du palisson de type 2. Expérimentations et clichés I. Sidéra. Dessins G. Der Aprahamian.

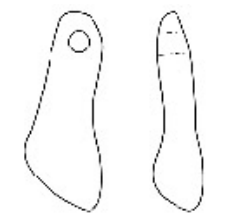

Stade d'usure 0 (théorique) Auc.Jn èmoussé : ébauche.
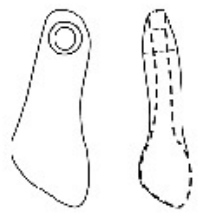

Stade d'usure $2(n=2)$ Totale disparition des traces de labrication.

Déformation de la perforation.

Amincissement sensible

de la paroi de la racine.

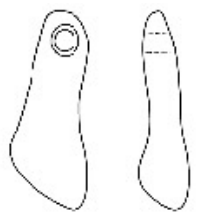

Stade d'usure $1(n-4)$

Emoussé de l'ensemble

de la dert:

sur races, burds el perloraliorr.

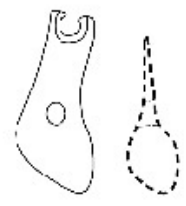

Stade d'usure 3 (théorique)

La paroi de la racine devient si fine qu elle se brise

au niveau de la perforation.

Un nouveau forage est exécuté.

Fig. 7 - Chaîne d'usure réalisée à partir d'une série de perles archéologiques perforées sur canines de cerf. D'après Sidéra et Giacobini, 2002, fig. 10, p. 224. 


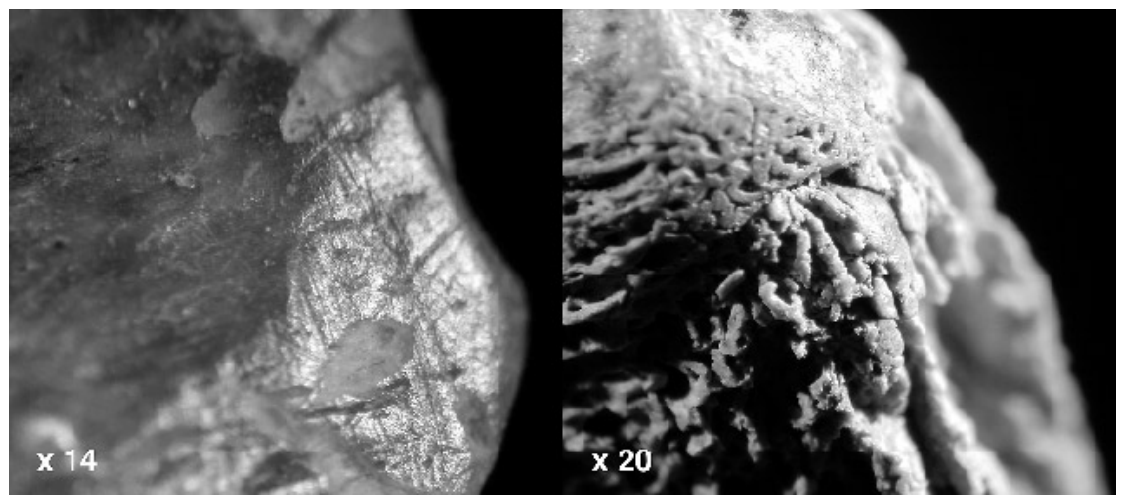

Fig. 8 - Diverses manifestations macroscopiques issues d'outils tranchants de Drama (Bulgarie, fouilles Pr Lichardus, université de Sarrebruck). $\mathrm{N}^{\circ} 1$ : enlèvements, émoussés, poli et stries $; \mathrm{n}^{\circ} 2$ : écrasements avec séparation des fibres osseuses. Clichés I. Sidéra (étude en cours).

nombreuses, hétérogènes, parallèles, qui se superposent à celui des surfaces et se développent depuis les bords vers l'intérieur des pièces (fig. 2 et 3 ).

Pour conclure, l'examen macroscopique est efficace sur des objets très usés, qui montrent des altérations de volume et des traces d'utilisation de surface bien développées. Pour exemple, les objets de parures ont un usage long et sont par conséquent très déformés. Les outils tranchants ont non seulement un long emploi mais aussi des traces d'utilisation bien développées parce qu'ils sont très actifs. Cet examen n'est cependant pas toujours suffisant pour préciser, dans certains cas, ni la matière mise en œuvre (par exemple, préciser la nature exacte des matières dures ou celle des matières souples), ni l'état de celle-ci, pour situer un outil donné dans une chaîne opératoire. De même, cet examen n'est pas toujours pertinent devant des outils peu déformés, en particulier la grande majorité des pointes où les indices fonctionnels macroscopiques sont faibles. Une méthodologie nouvelle et appropriée qui détaille les surfaces doit être mise en œuvre. Elle permettra de décrypter sur des objets «muets» sur le plan macroscopique, les matières sur lesquelles ils ont agi et leur action, ou d'optimiser l'identification déjà fournie par l'approche macroscopique. Il est difficile, avec les seules informations macroscopiques, de préciser si les estèques ont agi sur matière molle ou partiellement sèche. C'est l'analyse plus exhaustive des surfaces qui permet d'obtenir cette information importante du moment effectif d'emploi de l'outil dans la chaîne opératoire du façonnage d'un pot. Si les instruments en os employés pour la poterie existent en effet dans toutes les cultures néolithiques, leurs morphologies et leur action sur la matière diffèrent selon chacune (cf. ceux décrits par Binder et al. 1994; Martineau et Maigrot, 2004; Meneses Fernandez, 1994; Sidéra, 1993a; Sidéra et Giacobini, 2002; Sidéra et Claustre, à paraître).

Les limites de l'examen macroscopique résident pour le moment ici. L'attribution fonctionnelle qu'il permet d'acquérir est incomplète. Ajoutons pour finir que l'appareil ne permet pas de prendre des mesures exactes mais approximatives, ce qui interdit de mesurer les microphénomènes de surface et exige pour le faire l'emploi d'un autre appareillage dont la calibration est plus exacte.

\section{Altérations de surface, examen microscopique}

Un microscope optique à réflexion est employé ici pour poursuivre l'analyse et détailler les surfaces usées. Selon un protocole consensuel, les grossissements sont fixes, à l'exemple de ceux utilisés par les lithiciens : $100 \times$ et $200 \times$. L'usage du microscope à balayage, combiné à l'analyse chimique de résidus (Aimar et al., 1998; d'Errico et al., 1995; Lemoine, 1997 ; Stordeur et Anderson-Gerfaud, 1985) est également très efficace, mais l'accès limité et coûteux de ce type d'appareil fait que nous lui préférons une méthode courante, moins dispendieuse. Depuis deux ans, l'équipement avec lequel nous travaillons est constitué d'un microscope optique à réflexion (Nikon éclipse ME600) relié à un ordinateur qui permet d'acquérir des images par le biais d'une camera numérique (Axiocam, logiciel KS300, Zeiss) (équipement de la maison de l'Archéologie et de l'Ethnologie René Ginouvès) (fig. 9).

La pertinence de l'analyse microscopique de surface sera illustrée ici au travers de deux poinçons expérimentaux, répliques d'outils issus du site précéramique de Khirokitia (Chypre) (Stordeur, 1984; Legrand, 2003 et 2005a). L'un des deux poinçons a été utilisé pour perforer une peau fraîche de mouton (outil 1), l'autre pour perforer de l'écorce humide de chêne (outil 2). La durée d'utilisation respective des deux poinçons est de 65 et de $10 \mathrm{mn}$. Dans les deux cas, la perforation a été exécutée par percussion indirecte au percuteur dur. Les épiphyses ont constitué les plans de frappe (fig. $10, \mathrm{n}^{\circ} 1$ ).

La description des microphénomènes d'usure est inspirée des travaux de Rozalia Christidou, dont la démarche, au travers de la systématisation de l'emploi du microscope, est une contribution majeure à l'élaboration des principes de la microtracéologie osseuse 


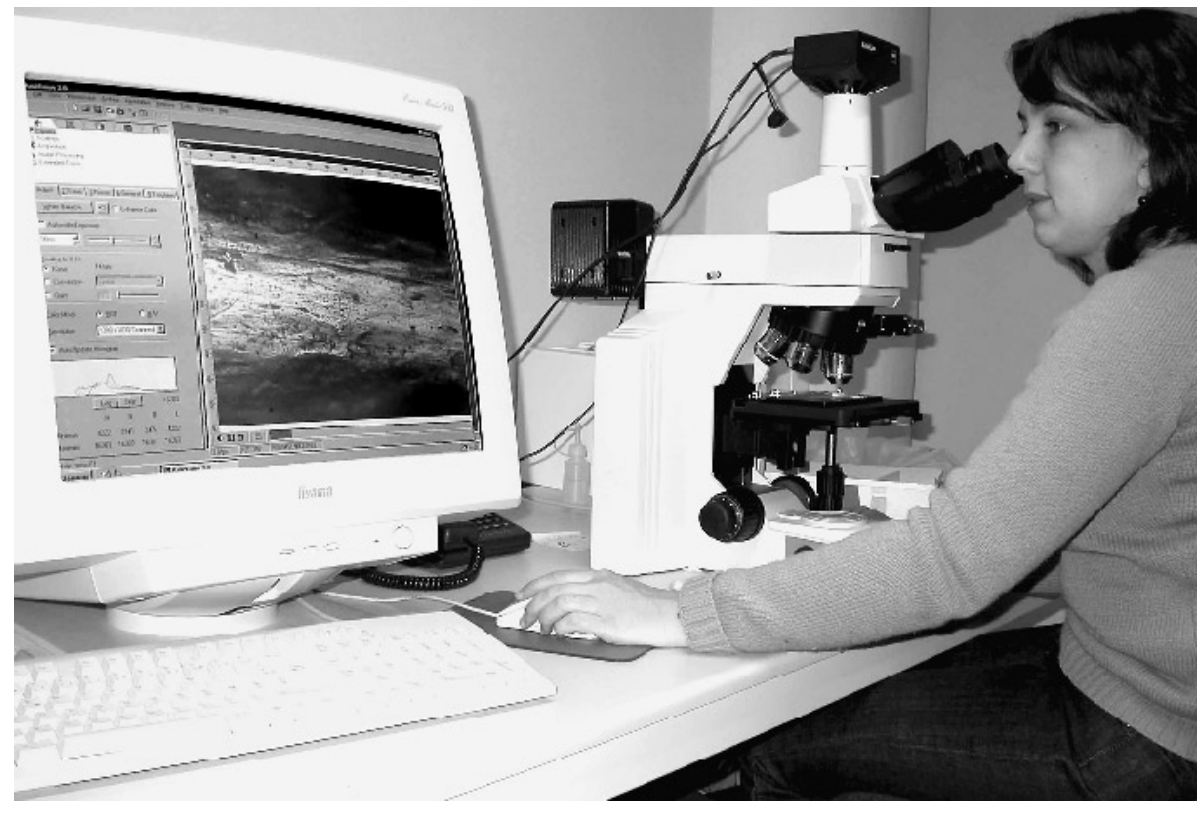

Fig. 9 - Équipement de microscopie avec capture et traitement d'image. Photo I. Sidéra.

(1999). Sa méthode, principalement axée sur l'appréhension du développement de l'usure par l'étude de la topographie des surfaces, s'intéresse aux élévations et aux creux, à leur fréquence, leur forme, leur texture, leur aspect et à leurs dimensions. La nature du poli (trame, coalescence, structure) est considérée comme un critère secondaire. Ainsi, lorsque nous parlons de poli, tel que cela a été défini pour la macroscopie, nous désignons seulement un état de surface brillant, signe d'usure, dont seules la présence, la localisation et l'extension sont prises en compte (Sidéra, 1993a, p. 202).

Commençons par décrire l'usure globale des deux poinçons afin de bien justifier l'apport de la microtracéologie. À l'œil nu et à un grossissement de $15 \times$, les pointes des deux poinçons montrent :

- un émoussé et un poli verni équivalents;

- un développement de l'usure similaire, dégressif et séquencé en trois zones, établi d'après le degré de l'émoussé des traces de fabrication.

La zone 1 d'usure concerne l'apex. Aucune trace de fabrication n'apparaît avant $2 \mathrm{~mm}$ sur l'outil 1 et avant $7 \mathrm{~mm}$ sur l'outil 2. De nombreuses stries d'utilisation longitudinales et des dépressions non linéaires sont déjà visibles à l'échelle macroscopique $(32 \times)$ dans les deux cas (fig. 10, $\mathrm{n}^{\circ} 2$ et $11, \mathrm{n}^{\circ} 2$ ). La zone 2 mesure $20 \mathrm{~mm}$ de long sur l'outil 1 et $4 \mathrm{~mm}$ de long sur l'outil 2. Sur les deux outils, les mêmes stries d'utilisation et les mêmes dépressions non linéaires de la zone 1 sont retrouvées sur la zone 2. En revanche, les traces de fabrication viennent à apparaître mais elles sont émoussées (fig. $10, n^{\text {os }} 3$ et $11, n^{\circ} 3$ ). La zone d'usure 3 , longue de $15 \mathrm{~mm}$ environ sur l'outil 1 et de $8 \mathrm{~mm}$ sur l'outil 2, laisse apparaître plus clairement encore les stries de fabrication. Celles-ci sont peu affectées par l'usure (fig. 10, n 4 et $11, n^{\circ} 4$ ).
Le séquençage de l'usure en différentes zones s'avère être une méthode efficace, qui l'est encore plus particulièrement sur les outils pointus. Il est ainsi permis de dissocier, dans le cas des usures les plus développées et claires, la zone de l'impact direct de l'outil dans la matière travaillée (zone 1), de la zone de frottement intermédiaire (zone 2) et de celle de l'extension maximale du frottement (zone 3). L'extension et les traces que comporte chaque zone sont des variables liées à la matière travaillée et à la cinématique de l'outil. Ici, par exemple, l'étendue de l'usure est différente sur les deux outils. Elle est longue de $40 \mathrm{~mm}$ environ dans le cas de l'outil 1 , de $19 \mathrm{~mm}$ pour l'outil 2 (fig. $10, \mathrm{n}^{\circ} 1$ et $11, \mathrm{n}^{\circ} 1$ ). Cette différence de longueur est la réponse du comportement de chaque matière considérée à l'action des outils. La peau, qui est une matière souple et élastique, enveloppe et épouse la morphologie de l'outil qui la travaille, d'où une usure d'étendue envahissante. Au contraire, l'écorce, qui est un matériau rigide, en limite la pénétration, d'où une usure d'étendue modérée.

À fort grossissement, l'usure en trois zones est toujours observée mais elle apparaît de façon plus différenciée sur chaque poinçon. Examinons tout d'abord 1'outil 1. À un grossissement de $100 \times$, la topographie est qualifiée. Stries, microtrous et cratères, les dépressions qui génèrent la topographie sont caractérisées. Si leur variété morphométrique et leur nombre sont élevés, comme dans le cas de la zone d'usure 1, la topographie est irrégulière. À $200 \times$, on observe le microrelief et on détaille les dépressions. L'usure des élévations et celle des bords et du fond des dépressions sont appréciées. Cela permet, pour le moins, de préciser à quelle nature de matériau on a affaire : matière dure ou matière souple. Sur l'outil 1, par exemple, le microrelief est homogène et, par endroit, régulier. Les 
élévations présentent un aspect bombé, elles sont vernies et de texture grenue (fig. $10, n^{\circ} 2$ ). Les stries sont courtes ou longues, superficielles ou profondes, continues, parallèles entre elles et parfois entrecroisées. Leur largeur varie de $1 \mu$ à $3 \mu$. Les bords des stries les plus larges sont émoussés et leur fond est partiellement lissé. Les cratères, dont la largeur varie de 9 à $27 \mu$, ont des bords émoussés et un fond rugueux. Les microtrous sont nombreux.

La morphologie, la distribution, la direction et les dimensions des dépressions de la zone 2 d'usure sont assez similaires $(100 \times)\left(\right.$ fig. $\left.10, n^{\circ} 3\right)$. En revanche, une légère différence du microrelief est observée, due à la présence des stries de fabrication $(200 \times)$. Le lissage de la zone 1 ne se reporte pas sur le microrelief de la zone 2, qui est seulement homogène. Le fond de certaines stries de fabrication est affecté par l'usure. Cratères et microtrous sont également plus nombreux que dans la zone 1 (fig. 10, $\mathrm{n}^{\circ} 3$ ).
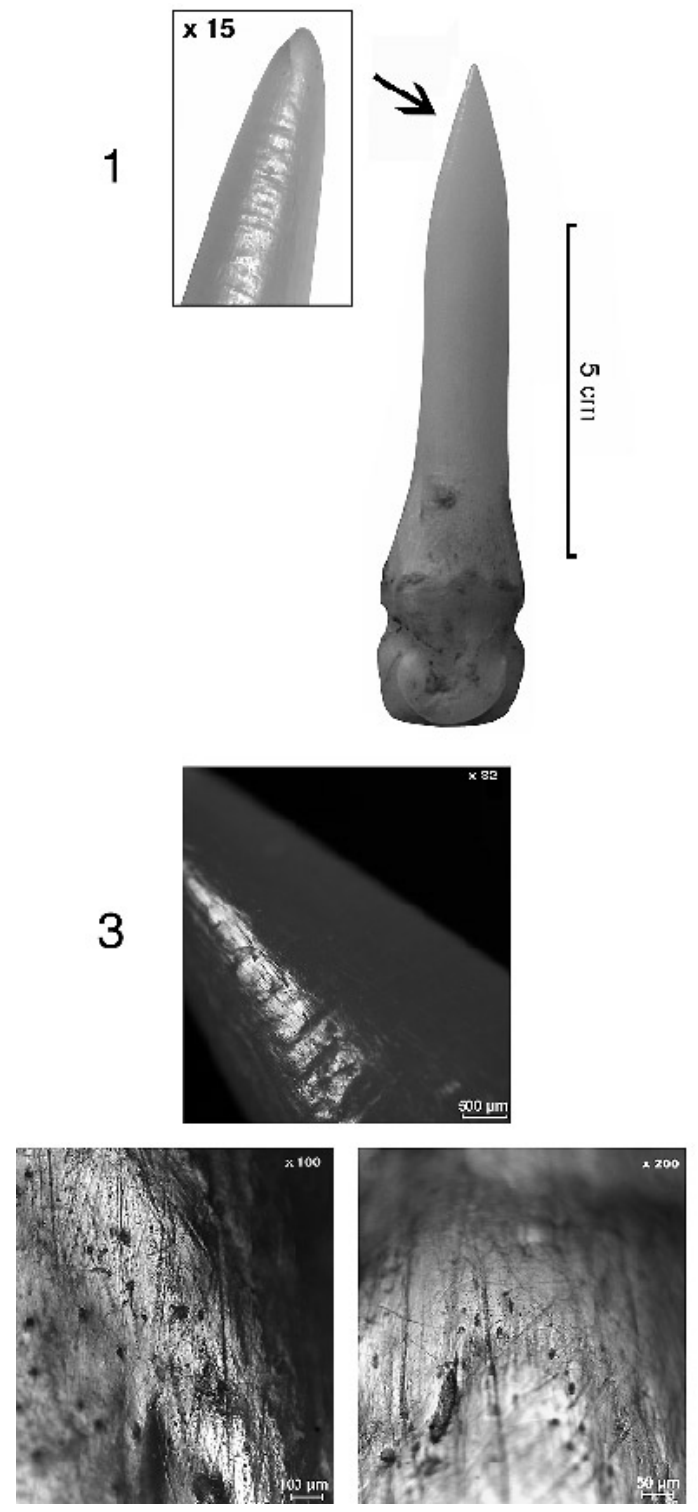

Fig. 10 - Caractères macroscopiques et microscopiques du poinçon expérimental $n^{\circ} 1$, issus du travail de la peau fraîche. $\mathrm{N}^{\circ} 1$ : vue du poinçon et de la pointe; $n^{\circ} 2$ : première zone d'usure; $n^{\circ} 3$ : deuxième zone d'usure; $n^{\circ} 4$ : troisième zone d'usure. Expérimentations et clichés A. Legrand (étude en cours).

Les stries de fabrication apparaissent très clairement sur la zone 3 d'usure et montrent un fond rugueux $(200 \times)$. Seules les élévations sont affectées par l'usure. Elles ont toujours le même aspect bombé et la même texture grenue. Leur brillance est, en revanche, atténuée. Les cratères ont disparu. Quant aux stries d'utilisation, elles ne sont disposées que sur les élévations. Elles diffèrent de celles des deux zones précédentes en ce qu'elles sont strictement parallèles entre elles, non plus entrecroisées, et beaucoup plus ordonnées et serrées. Elles ont cependant toujours les mêmes dimensions (fig. 10, $\mathrm{n}^{\circ} 4$ ).

Abordons maintenant l'examen de l'outil 2. Avec un grossissement de $100 \times$, la zone 1 présente une topographie régulière. À 200 ×, le microrelief est régulier. La surface est vernie et recoupée par de fréquents microtrous et une forte densité de stries unidirectionnelles, fines (inférieures à $1 \mu$ ), longues, superficielles, continues ou discontinues (fig. 11, $\mathrm{n}^{\circ} 2$ ). Quelques
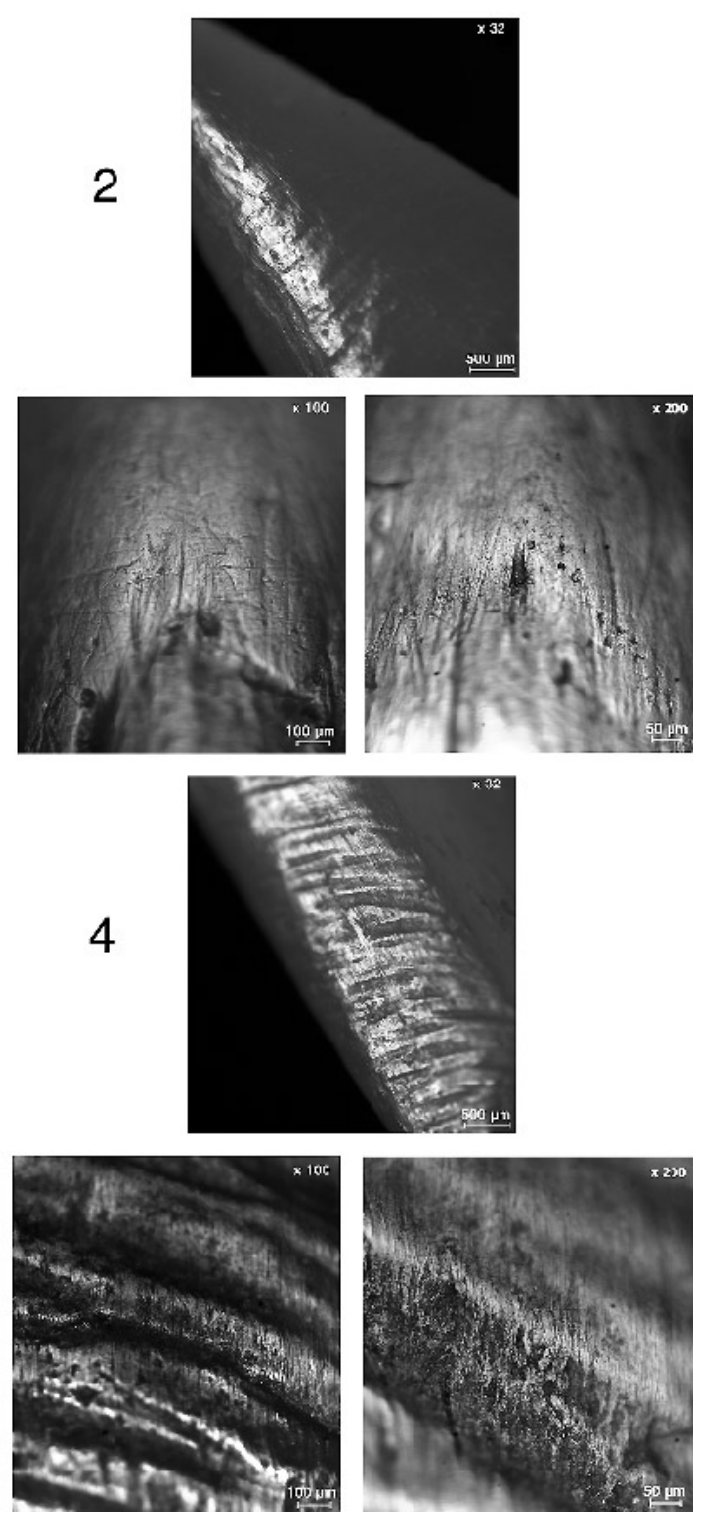
stries transversales, superficielles et fines, apparaissent. Les cratères présentent des dimensions variées, de $9 \mu$ à $34 \mu$. Leurs bords sont toujours émoussés et leur fond est rugueux ou partiellement lissé.

Sur la zone 2, l'aspect de la topographie est similaire, en revanche, le microrelief est homogène (fig. 11, $n^{\circ} 3$ ). Les cratères, aux bords toujours émoussés et au fond toujours rugueux, sont plus nombreux que dans la zone 1 .

Sur la zone 3, la topographie est proche de celle qu'elle était juste après le façonnage. Les stries de fabrication sont presque intactes. Elles sont rugueuses, profondes et larges, associées à de larges dépressions qui couvrent la majeure partie de la zone (fig. 11, $\left.n^{\circ} 4\right)$. Le microrelief est homogène. Les élévations sont faiblement émoussées. Leur aspect est plat, leur texture est toujours grenue. Leur brillance est atténuée, en comparaison de la zone 2. Elles montrent aussi les mêmes stries fines d'utilisation. Les cratères disparaissent. En revanche, les microtrous restent fréquents.

Grâce à un niveau de détail bien supérieur, l'usure qui apparaissait relativement homogène sur les deux pointes à l'échelle macroscopique se révèle bien différenciée à l'échelle microscopique. Si l'on se fonde sur la zone d'usure 1, où les traces sont les plus développées, la topographie et le microrelief montrent des différences significatives entre les deux pointes. Le travail de la peau montre une topographie irrégulière et un microrelief principalement homogène. Le travail de l'écorce de chêne se caractérise par une topographie régulière et un microrelief régulier. Les propriétés du matériau travaillé, et notamment leur capacité de déformation, sont déterminantes sur la formation de ces traces. Elles sont aussi déterminantes pour le degré d'envahissement de l'usure. D'autres différences complètent les premières. Elles concernent la morphologie, les dimensions, la direction et la fréquence des stries et des dépressions non linéaires. Malgré un même mode d'action, une variabilité directionnelle et morphométrique importante entre les deux outils peut s'observer. La perforation de la peau produit des stries de taille et de forme variées. Elles sont, en outre, pluridirectionnelles. Au contraire, dans le cas de la perforation d'écorce humide, les stries sont plus homogènes et bien plus ordonnées dans l'axe de la pièce.
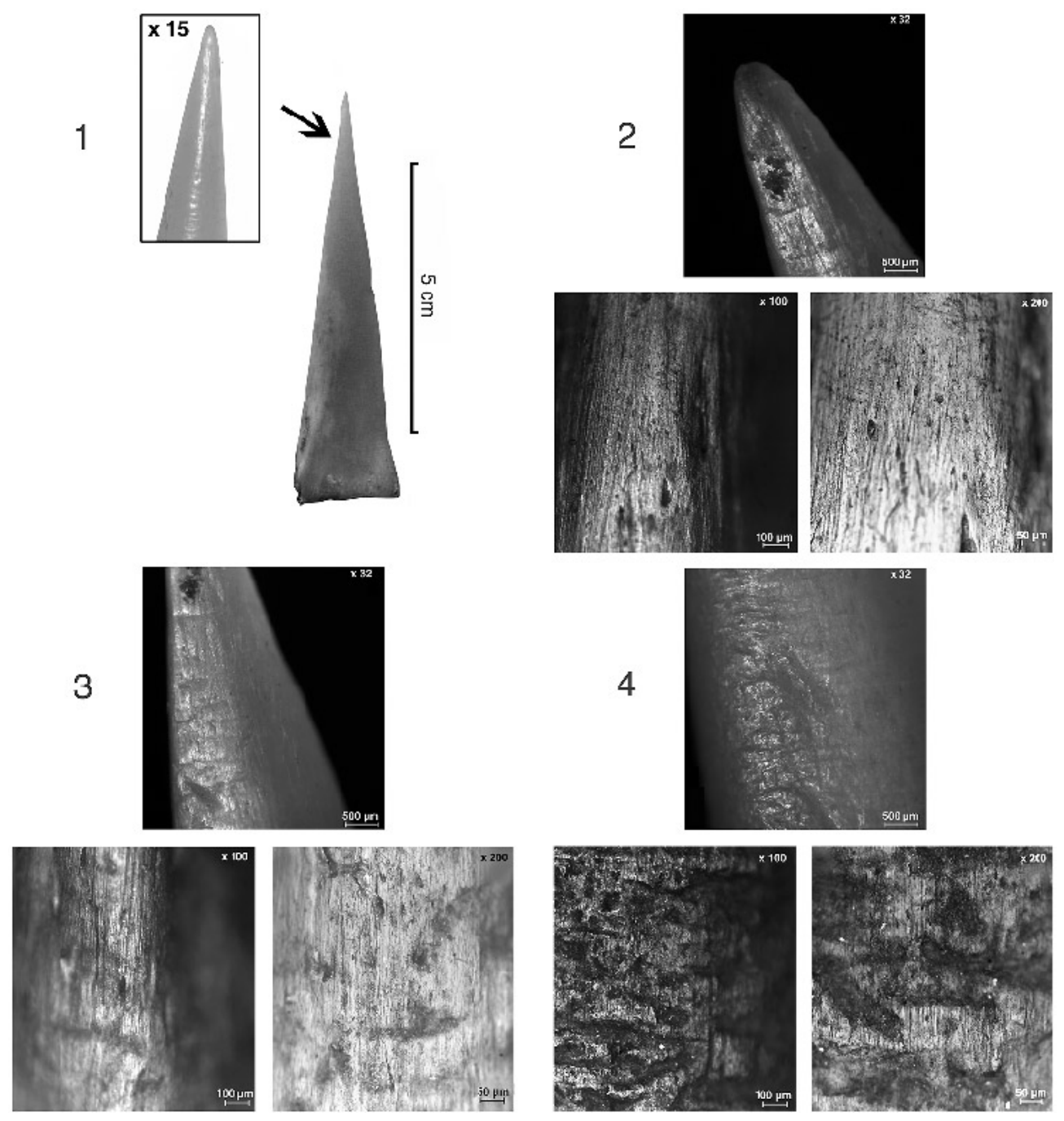

Fig. 11 - Caractères macroscopiques et microscopiques du poinçon expérimental $\mathrm{n}^{\circ} 2$, issus du travail de l'écorce humide. $\mathrm{N}^{\circ} 1$ : vue du poinçon et de la pointe; $\mathrm{n}^{\circ} 2$ : première zone d'usure; $\mathrm{n}^{\circ} 3$ : deuxième zone d'usure; $n^{\circ} 4$ : troisième zone d'usure. Expérimentations et clichés A. Legrand (étude en cours). 
C'est une succession de cet ordre de caractères microscopiques qui permet d'identifier la matière mise en œuvre, son état et la cinématique correspondant au travail de l'outil. Le travail en cours vise à aboutir à une typologie de l'usure, en référence aux actions et aux matières travaillées (Legrand, 2005a).

\section{CONCLUSION}

L'identification fonctionnelle des objets en matière osseuse n'est pas simple. L'information est extraite au terme d'une analyse lente et progressive, un protocole d'examens macro- puis microscopiques chaînés et continus, basé sur l'expérimentation. De très nombreux critères doivent être pris en compte. C'est le processus optimal.

Quelques certitudes ont été acquises, qui permettent de poser des fondements analytiques fiables. Nous savons aujourd'hui, ayant concrètement éprouvé ses principes, que la méthodologie mise en œuvre, même si elle est perfectible, est établie sur des critères opératoires. L'analyse macroscopique, principalement confrontée aux altérations du volume des objets et donc aux objets marqués par un degré d'usure développé, permet des identifications fonctionnelles fiables mais imparfaites. L'analyse microscopique, qui s'intéresse davantage aux altérations de surface, complète la précédente. Elle est plus particulièrement pertinente pour des usures diffuses et des volumes initiaux peu déformés, soit pour tous les objets dont l'usure résulte principalement de la friction, comme les poinçons, les aiguilles et les crochets. L'appréhension des usures de surface, microreliefs et dépressions, dont l'observation est optimale au microscope, est nécessaire pour préciser les matières avec lesquelles ces objets ont été en contact.

Malgré ces acquis, il convient de continuer le travail afin de fiabiliser davantage encore la démarche. La compréhension des phénomènes à l'origine des usures répertoriées est notamment à affiner, grâce à des recherches plus précises et systématiques. Seules, l'intensification et la structuration de l'expérimentation, issues d'une coopération entre chercheurs, permettront de progresser significativement. Comprendre les interactions entre les caractères macroscopiques et microscopiques de l'usure conduira, à terme, à un modèle analytique qui permettra d'identifier la majorité des fonctions des artefacts. Du moins l'espérons-nous.

Ajoutons pour finir que l'informatique a changé considérablement notre façon de travailler. Elle permet de quantifier les microphénomènes de surface, de faciliter et de multiplier la capture des images et des mesures puis de les traiter par voie statistique. À terme, des banques d'images et de données numériques pourront être fournies. Pour le plus grand profit de cette sous-discipline, l'informatique simplifie également l'échange des données et favorise, du coup, leur transmissibilité. Précisons qu'il est évident que la méthode exposée ici peut être complétée par d'autres types d'approches, notamment chimiques et tribologiques.

\section{RÉFÉRENCES BIBLIOGRAPHIQUES}

AIMAR A., CILLI C., GIACOBINI G., MALERBA G. (1998) - Scanning Electron Microscopy at bone surface modification. A Contribution to the Study of Anthropic Actions on Animal Hard Tissues from Archeological Sites, Science and Technology for the Safeguard of Cultural Heritage in the Mediterranean Basin, éd. du CNRS, Rome, p. $1437-1443$.

ANDERSON P. (1992) - Préhistoire de l'agriculture. Nouvelles approches expérimentales et ethnographiques, Monographie du CRA, $\mathrm{n}^{\circ} 6$, éd. du CNRS, Paris, 403 p.

AVERBOUH A. (2000) - Technologie de la matière osseuse travaillée et implications palethnologiques. L'exemple des chaînes d'exploitation du bois de cervidés chez les Magdaléniens des Pyrénées, thèse de doctorat de l'université Paris I, 503 p.

AVERBOUH A., BODU P. (2002) - Fiche percuteur sur partie basilaire de bois de cervidé, in M. Patou-Mathis dir., Fiches typologiques de l'industrie osseuse préhistorique, Cahier X : compresseurs, percuteurs, retouchoirs, éd. de la Société préhistorique française, Paris, p. 117-131.

BARGE H. (1982) - Les perles-pendeloques à coches en os. Reconstitution expérimentale, in H. Camps-Fabrer dir., Industrie de l'os néolithique et de l'Âge des Métaux, $\mathrm{n}^{\circ}$ 2, éd. du CNRS, Paris, p. 113123.

BILLAMBOZ A. (1977) - L'industrie en bois de cerf en Franche-Comté au Néolithique et au début de l’Âge du Bronze, Gallia Préhistoire. $\mathrm{n}^{\circ} 20$, fasc. 1 , p. 91-176.

BINDER D., GASSIN B., SÉNÉPART I. (1994) - Éléments pour la caractérisation des productions céramiques néolithiques dans le Sud de la France, Actes du colloque "Terre cuite et société. La céramique, document technique, économique, culturel », éd. Association pour la Diffusion des Connaissances en Archéologie, Juan-les-Pins, p. $255-$ 267.
BONNARDIN S. (2004) - La parure funéraire du Néolithique ancien en Bassins parisien et rhénan. Matériaux, techniques, fonctions et usage social, thèse de doctorat de l'université Paris I.

CAMPANA D.V. (1989) - Natufian and Protoneolithic Bone Tools. The Manufacture and Use of Bone Implements in the Zagros and the Levant, British Archaeological Reports, International Series, no 494, Oxford, $156 \mathrm{p}$.

CAMPS-FABRER H. (1966) - Matière et art mobilier dans la Préhistoire nord-africaine et saraharienne, Mémoires du Centre de Recherches anthropologiques, préhistoriques et ethnographiques d'Alger, $\mathrm{n}^{\circ} 5$, Paris.

CAMPS-FABRER H., D'ANNA A. (1977) - Fabrication expérimentale d'outils à partir de métapodes de moutons et de tibias de lapins, in H. Camps-Fabrer dir., Méthodologie appliquée à l'industrie de l'os préhistorique, Actes du deuxième colloque international sur l'industrie de l'os dans la Préhistoire, abbaye de Sénanque, juin 1976, éd. du CNRS, Paris, p. 33-57.

CHIQUET P., PÉTREQUIN P., RACHEZ E. (1997) - Les défenses de sanglier, in P. Pétrequin dir., Les sites littoraux néolithiques de Clairvaux-les-Lacs et de Chalain (Jura), 3 (2), Chalain station 3, 3200 av. $J .-C$., éd. de la Maison des sciences de 1'Homme, Paris, p. 511-521.

CHRISTENSEN M. (1996) - Le travail et l'usage de l'ivoire au Paléolithique supérieur, tracéologie des outils en silex et caractérisation chimique des polis d'utilisation, thèse de doctorat de l'université Paris I, $325 \mathrm{p}$.

CHRISTIDOU R. (1999) - Outils en os néolithiques du Nord de la Grèce. Étude technologique, thèse de doctorat de l'université Paris X-Nanterre, $418 \mathrm{p}$.

CHRISTIDOU R. (2001) - Usure frontale d'un outil tranchant en os : deux mouvements opposés, une séquence technique?, in 
L. Bourguignon, I. Ortega et M.-C. Frère-Sautot dir., Préhistoire et approche expérimentale, Préhistoires 5, éd. Monique Mergoil, Montagnac, p. 259-266.

CHRISTIDOU R. (2004) - Fabrication d'outils en os. Exemple d'opérations de raclage et d'abrasion, in L. Astruc et R. Vargiolu coord., Dossiers d'Archéologie, ${ }^{\circ}$ 290, numéro spécial : la tribologie. Comment la science fait parler les vestiges archéologiques, p. 5657.

CHRISTIDOU R., LEGRAND A. (2005) - Hide working and bone tools: experimentation design and applications, in H. Luik, A.M. Choyke, C.E. Batey et L. Lougas dir., From Hooves to horns, from mollusc to mammoth. Manufacture and use of bone artifacts from Prehistoric Times to the Present, Proceedings of the $4^{\text {th }}$ Meeting of the (ICAZ) Worked Bone Research Group, Tallinn (Estonie), 26-31 août 2003, Muinasaja Teadus, $\mathrm{n}^{\circ} 15$, p. $385-396$

CRISTIANI E., ALHAIQUE F. (2005) - Flint $v s$ metal: the manufacture of bone tools in the Eneolithic site of Conelle di Arcevia (central Italy), in H. Luik, A.M. Choyke, C.E. Batey et L. Lougas dir., From Hooves to horns, from mollusc to mammoth. Manufacture and use of bone artifacts from Prehistoric Times to the Present, Proceedings of the $4^{\text {th }}$ Meeting of the (ICAZ) Worked Bone Research Group, Tallinn (Estonie), 26-31 août 2003, Muinasaja Teadus, $n^{\circ} 15$, p. 397-403.

DAUVOIS M. (1974) - Industrie osseuse préhistorique et expérimentations, in H. Camps-Fabrer dir., Actes du premier colloque international sur l'industrie de l'os dans la Préhistoire, abbaye de Sénanque, avril 1974, Publications de l'université de Provence, p. 73-84.

DAVID E. (1999) - L'industrie en matière dure d'origine animale du Mésolithique ancien en Europe du Nord: contribution de l'analyse technologique à la définition du Maglemosien, thèse de doctorat de l'université Paris X-Nanterre.

DAVID E. (2003) - Contribution de la technologie osseuse à la définition du Maglemoisien (Mésolithique ancien de l'Europe du Nord), in M. Patou-Mathis, P. Cattelain et D. Ramseyer coord., L'industrie osseuse pré- et protohistorique en Europe. Approches technologiques et fonctionnelles, Actes du colloque 1.6, XIV congrès de l'UISPP Liège, septembre 2001, Bulletin du Cercle archéologique HesbayeCondroz, t. XXVI, p. 75-86.

D'ERRICO F. (1991) - Étude technologique à base expérimentale des entailles sur matière dure animale. Implications pour l'identification de systèmes de rotation, Expérimentations en archéologie : bilan et perspectives, Actes du colloque international de Beaune, avril 1988 t. 2 : La terre. L'os et la pierre, la maison et les champs, éd. Errance, Paris, p. 83-97.

D'ERRICO F. (1993) - Identification des traces de manipulation, suspension, polissage sur l'art mobilier en os, bois de cervidé, ivoire, in P.C. Anderson, S. Beyries, M. Otte et H. Plisson dir., Traces et fonction, les gestes retrouvés, Actes du colloque international de Liège, décembre 1990, vol. 1, ERAUL, nº 50, p. 177-188.

D'ERRICO F. (1996) - Image analysis and 3-D optical surface profiling of upper Palaeolithic mobiliary art, Microscopy and Analysis, p. 27

D'ERRICO F., GIACOBINI G. (1985) - Approche méthodologique de l'analyse de l'outillage osseux : un exemple d'étude, L'Anthropologie, t. 89 , fasc. 4 , p. $457-472$.

D'ERRICO F., GIACOBINI G., HATHER J., POWER-JONES A.H., RADMILI A.M. (1995) - Possible bone treshing tools from the Neolithic levels of Grotta dei Pïccioni (Abruzzo, Italy), Journal of Archaeological Science, $\mathrm{n}^{\circ} 22$, p. 537-549.

D'ERRICO F., VILLA P. (1997) - Holes and grooves: the contribution of microscopy and taphonomy to the problem of art origins, Journal of Human Evolution, $\mathrm{n}^{\circ} 33$, p. 1-31.

ETTOS (1985) - Techniques de percussion appliquées au matériau osseux, premières expériences, Cahiers de l'Euphrate, $\mathrm{n}^{\circ} 4$, éd. de la Recherche sur les civilisations, Paris, p. 373-381.

ETTOS (1991) - Des chasse-lames en os? Une étude expérimentale, Expérimentations en archéologie : bilan et perspectives, Actes du colloque international de Beaune, avril 1988, t. 2 : La terre. L'os et la pierre, la maison et les champs, éd. Errance, Paris, p. 63-73.
GOUTAS N. (2004) - Caractérisation et évolution du Gravettien en France par l'approche techno-économique des industries en matières dures animales (étude de six gisements du Sud-Ouest), thèse de doctorat de l'université Paris I.

LEGRAND A. (2000) - Vers une identification technologique et fonctionnelle des outils biseautés en matière osseuse : le site magdalénien de la Garenne, mémoire de DEA, université Paris I.

LEGRAND A. (2003) - Concordance des formes et des fonctions ? Étude technofonctionnelle des poinçons en os de Khirokitia (Néolithique précéramique, Chypre), Préhistoire et Anthropologie méditerranéennes, t. 12, p. 189-196.

LEGRAND A. (2005a) - Nouvelle approche méthodologique des assemblages osseux du Néolithique de Chypre. Entre technique, fonction et culture, thèse de doctorat de l'université Paris I, 698 p.

LEGRAND A. (2005b) - A new evidence on the bone reduction techniques from Khirokitia - Cyprus ( $7^{\text {th }}$ millennium cal. B.C.), in H. Luik, A.M. Choyke, C.E. Batey et L. Lougas dir., From Hooves to horns, from mollusc to mammoth. Manufacture and use of bone artifacts from Prehistoric Times to the Present, Proceedings of the $4^{\text {th }}$ Meeting of the (ICAZ) Worked Bone Research Group, Tallinn (Estonie), 26-31 août 2003, Muinasaja Teadus, $\mathrm{n}^{\circ} 15$, p. 385-396.

LEMOINE G. (1997) - Use Wear on Bone and Antler Tools from the Mackensie Inuit, British Archaeological Reports, International Series, $\mathrm{n}^{\circ} 679$, Oxford, $130 \mathrm{p}$.

LIOLIOS D. (1999) - Variabilité et caractéristiques des matières osseuses au début de l'Aurignacien, thèse de doctorat de l'université Paris X-Nanterre, 359 p.

MAIGROT Y. (1997) - Tracéologie des outils tranchants en os des Ve et $\mathrm{IV}^{\mathrm{E}}$ millénaires av. J.-C. en Bassin parisien. Essai méthodologique et application, Bulletin de la Société préhistorique française, t. 94, $\mathrm{n}^{\circ} 2$, p. $198-216$.

MAIGROT Y. (2001) - Le débitage du bois de cerf au Néolithique fina à Chalain et Claivaux (Jura, France). Approche expérimentale, in L. Bourguignon, I. Ortega et M.-C. Frère-Sautot dir., Préhistoire et approche expérimentale, Préhistoires 5, éd. Monique Mergoil, Montagnac, p. 165-172.

MAIGROT Y. (2003) - Étude technologique et fonctionnelle de l'outillage en matières dures animales. La station 4 de Chalain (Néolithique final, Jura, France), thèse de doctorat de l'université Paris I, 284 p.

MARTINEAU R., MAIGROT Y. (2004) - Les outils en os utilisés pour le façonnage des poteries néolithiques de la station 4 de Chalain (Jura, France), in P. Bodu et C. Constantin dir., Approches fonctionnelles en Préhistoire, Actes du $X X V^{p}$ congrès préhistorique de France, Nanterre, novembre 2000, éd. de la Société préhistorique française, Paris, p. 83 96.

MENESES FERNANDEZ M.D. (1993) - Reconstruccion technica, experimentacion y estudio comparativo de los 'tensadores textiles' de hueso del neolitico y Calcolitico en Andalucia (Espana), in P.C. Anderson, S. Beyries, M. Otte et H. Plisson dir., Traces et fonction, les gestes retrouvés, Actes du colloque international de Liège, décembre 1990, vol. 1, ERAUL, nº 50, p. 309-316.

MENESES FERNANDEZ M.D. (1994) - Utiles de hueso del Neolitico final del sur de la Peninsula Iberica empleados en alfareria : placas curvas, biseles, placas y apuntados, Trabajos de Prehistoria, t. 51, fasc. 1, p. 143-156

NANDRIS J.-G., CAMPS-FABRER H. (1993) - Fiche 6.3 : cuillers à cuilleron à base en V du FTN, in $\mathrm{H}$. Camps-Fabrer dir., Fiches typologiques de l'industrie osseuse préhistorique. Les éléments récepteurs, Cahier VI, éd. de l'Université de Provence, Aix-en-Provence, p. 153 162.

NEWCOMER M.H. (1977) - Experiments in upper Paleolithic bone work, in H. Camps-Fabrer dir., Méthodologie appliquée à l'industrie de l'os préhistorique, Actes du deuxième colloque international sur l'industrie de l'os dans la Préhistoire, abbaye de Sénanque, juin 1976. éd. du CNRS, Paris, p. 293-301.

OLSEN S.A. (1989) - On distinguishing Natural from Cultural damage on Archaeological Antler, Academic Press, n 16, p. 125-135. 
OLSEN S.A. (2001) - The importance of thong-smoothers at Botai, Kazakhstan, in A.M. Choyke et L. Bartosiewicz dir., Crafting bone: Skeletal Technologies through Time and Space, Proceedings of the $2^{\text {nd }}$ meeting of the Worked Bone Research Group (ICAZ), 31 août-5 septembre 1999, Budapest, British Archaeological Reports, International Series, no 937, Oxford, p. 197-206.

PELEGRIN J. (1991) - Aspects de la démarche expérimentale en technologie lithique, 25 ans d'études technologiques en Préhistoire, $11^{e s}$ rencontres internationales d'archéologie et d'histoire d'Antibes, éd. de l'Association pour la Promotion et la Diffusion des Connaissances archéologiques, Juan-les-Pins, p. 57-63.

PÉTILLON J.-M. (2004) - Des Magdaléniens en armes : technologie des armatures de projectiles en bois de cervidé du Magdalénien supérieur de la grotte d'Isturitz (Pyrénées-Atlantiques), thèse de doctorat de l'université Paris I.

PELTIER A. (1986) - Étude expérimentale des surfaces osseuses façonnées et utilisées, Bulletin de la Société préhistorique française, t. 83, fasc. 1, p. 5-7.

PELTIER A., PLISSON H. (1986) - Microtracéologie fonctionnelle sur l'os, quelques résultats expérimentaux, Outillage en os et en bois de cervidés II, Artefacts 3, éd. du Centre de Recherche et de Documentation archéologique de Viroinval (Belgique), Viroinval, p. 69-80.

PLISSON H. (1991) - Tracéologie et expérimentation; bilan d'une situation, Expérimentations en archéologie : bilan et perspectives, Actes du colloque international de Beaune, avril 1988, t. 2 : La terre. L'os et la pierre, la maison et les champs, éd. Errance, Paris, p. 152160.

POPLIN F. (1974) - Deux cas particuliers de débitage par usure, in H. Camps-Fabrer dir., Actes du premier colloque international sur l'industrie de l'os dans la Préhistoire, abbaye de Sénanque, avril 1974, éd. de l'université de Provence, Aix-en-provence, p. 85-92.

PROVENZANO N. (1999) - Techniques et procédés de fabrication des industries osseuses terramaricoles de l'Âge du Bronze, in M. Julien, A. Averbouh, D. Ramseyer, C. Bellier, D. Buisson, P. Cattelain, M. Pathou-Mathis et N. Provenzano dir., Préhistoire d'os. Recueil d'études sur l'industrie osseuse préhistorique offert à Henriette Camps-Fabrer, Aix-en Provence, p. 273-289.

RAMSEYER D. dir. (2005) - Fiches typologiques de l'industrie osseuse préhistorique, Cahier XI : Matières et techniques, éd. de la Société préhistorique française, Paris, $230 \mathrm{p}$.

SCHIBLER J. (2001) - Experimental Production of Neolithic Bone and Antler Tools, in A.M. Choyke et L. Bartosiewicz dir., Crafting bone: Skeletal Technologies through Time and Space, Proceedings of the $2^{\text {nd }}$ meeting of the Worked Bone Research Group (ICAZ), Budapest, 31 août-5 septembre 1999, British Archaeological Reports, International Series, $n^{\circ}$ 937, Oxford, p. 49-60.

SEMENOV S.A. (1964) - Prehistoric Technology, Cory, Adam et Mackay, Londres, 211 p.

SÉNÉPART I. (1991) - Industrie osseuse et traitement thermique. Compte rendu de quelques expérimentations, Expérimentations en archéologie : bilan et perspectives, Actes du colloque international de Beaune, avril 1988, t. 2, La terre. L'os et la pierre, la maison et les champs, éd. Errance, Paris, p. 49-55.

SIDÉRA I. (1989) - Un complément des données sur les sociétés rubanées, l'industrie osseuse de Cuiry-lès-Chaudardes, British Archaeological Reports, International Series, n 520, Oxford, 163 p.

SIDÉRA I. (1993a) - Les assemblages osseux en Bassins parisien et rhénan du $\mathrm{VI}^{e}$ au IV millénaire B.C. Histoire, techno-économie et culture, thèse de doctorat de l'université de Paris I, 636 p.

SIDÉRA I. (1993b) - L'outillage lithique et osseux à Darion et à Cuirylès-Chaudardes. Une consécration aux matières animales, in P.C. Anderson, S. Beyries, M. Otte et H. Plisson dir., Traces et fonction, les gestes retrouvés, Actes du colloque international de Liège. décembre 1990, vol. 1, ERAUL, nº 50, p. 147-157.

SIDÉRA I. (1997) - Rapport d'étude de l'assemblage osseux de Drama (Bulgarie), Bericht der römisch-germanischen Kommission, $\mathrm{n}^{\circ} 77$ p. $120-129$.
SIDÉRA I. (2000) - Animaux domestiques, bêtes sauvages et objets en matières animales du Rubané au Michelsberg. De l'économie aux symboles, des techniques à la culture, Gallia Préhistoire, $\mathrm{n}^{\circ} 42$, p. $108-194$

SIDÉRA I. (2001) - Feu et industrie osseuse. Un marqueur d'identité culturelle, in A. Hauptmann dir., La pyrotechnologie à ses débuts. Évolution des premières industries faisant usage du feu, Paléorient, $\mathrm{n}^{\circ} 26$, fasc. 2 , p. 51-59 et pl. 6-7.

SIDÉRA I. (2003) - Rapport d'étude du mobilier en os de Mareuil-lèsMeaux «les Vignolles», s.1., 10 p.

SIDÉRA I. (2005) - Technical data, typological data: a confrontation, in H. Luik, A.M. Choyke, C.E. Batey et L. Lougas dir., From Hooves to horns, from mollusc to mammoth. Manufacture and use of bone artifacts from Prehistoric Times to the Present, Proceedings of the $4^{\text {th }}$ Meeting of the (ICAZ) Worked Bone Research Group, Tallinn (Estonie), 26-31 août 2003, Muinasaja Teadus 15, p. 81-90.

SIDÉRA I., CLAUSTRE F. (à paraître) - Identité technique et fonctionnelle des outils en os, en bois de cerf et dents de Montou, in F. Claustre dir., Les occupations de la grotte de Montou (Pyrénées-orientales), Documents d'Archéologie française, Paris.

SIDÉRA I., GIACOBINI G. (2002) - Outils, armes et parure en os funéraires à la fin du Néolithique, d'après Val-de-Reuil et Porte-Joie (Eure). Représentations individuelles et pratiques collectives, Gallia Préhistoire, $\mathrm{n}^{\circ} 44$, p. 215-230.

STORDEUR D. (1977) - La fabrication des aiguilles à chas, observation et expérimentation, in $\mathrm{H}$. Camps-Fabrer dir., Méthodologie appliquée à l'industrie de l'os préhistorique, Actes du deuxième colloque international sur l'industrie de l'os dans la Préhistoire, abbaye de Sénanque, juin 1976, éd. du CNRS, Paris, p. 251-256.

STORDEUR D. (1983) - Quelques remarques pour attirer l'attention sur l'intérêt d'une recherche commune entre tracéologues du silex et technologues de l'os, in M.-C. Cauvin dir., Traces d'utilisation sur les outils néolithiques du Proche Orient, Table ronde internationale, Travaux de la Maison de 1'orient, $n^{\circ}$ 5, éd. du CNRS, Paris, p. 231240.

STORDEUR D. (1984) - L'industrie osseuse de Khirokitia, in A. Le Brun dir., Fouilles récentes à Khirokitia (Chypre) 1977-1981, éd. Recherches sur les Civilisations, Paris, p. 129-162.

STORDEUR D. (1988) - Outils et armes en os de Mallaha, Mémoires et travaux du Centre de Recherche français de Jérusalem, $\mathrm{n}^{\circ} 6$, éd. Paléorient, Paris, $135 \mathrm{p}$.

STORDEUR D. (1989) - Des technologies nouvelles au service de la technologie? L'exemple des outils d'os préhistoriques, Notes et monographies techniques, $\mathrm{n}^{\circ}$ 25, éd. du CNRS, Paris, p. 127-150.

STORDEUR D., ANDERSON-GERFAUD P. (1985) - Les omoplates encochées néolithiques de Ganj-Dareh (Iran). Étude morphologique et fonctionnelle, Cahiers de l'Euphrate, no 4, p. 289-313.

TABORIN Y. (1993) - La parure en coquillage au Paléolithique, XXIX suppl. à Gallia Préhistoire, éd. du CNRS, Paris, 538 p.
Isabelle SIDÉRA

CNRS, UMR 7055-MAE

21, allée de l'Université, 92023 Nanterre cedex isabelle.sidera@mae.u-paris10.fr

Alexandra LEGRAND Post-doc, UMR 7041-MAE 21, allée de l'Université, 92023 Nanterre cedex a.legrand@free.fr 